\title{
Current driven spin-wave instability triggered by the anomalous Hall effect.
}

\author{
I. Ya. Korenblit ${ }^{1}$ \\ ${ }^{1}$ The Raymond and Beverly Sackler School of Physics and Astronomy, Tel Aviv University, Tel Aviv 69978, Israel
}

(Dated: October 26, 2018)

\begin{abstract}
We studied the effect of strong electric current on spin waves interacting relativistically with the current. The spin-wave spectrum is calculated at arbitrary direction of the wave vector. It is shown that the alternating Hall current generated by the alternating magnetic moment of the spin waves, reduces the spin-wave damping. At strong enough unpolarized dc current the damping changes sign, and the spin-wave amplitude starts to increase exponentially fast with time. The critical current for the spin-wave instability is determined mainly by the anomalous Hall effect, and can be much smaller than that for the spin-torque mechanism of instability.
\end{abstract}

PACS numbers: 75.30.Ds, 75.40.Gb, 75.47.-m

Keywords:

Current induced switching of the magnetization or spin wave excitation in magnetically inhomogeneous systems, e.g. multilayers, has received considerable attention during the last decade. [1] When a spin-polarized current passes through a ferromagnetic layer, the electrons transfer their spin angular momentum to the localized spins of the ferromagnet resulting in a spin torque acting on the magnetization. 2, 3] The theoretical predictions were confirmed by several groups, the experiments being performed mainly with trilayers of structure ferromagnetnormal metal-ferromagnet, in which the layer magnetization may be noncollinear, see Ref. 1 and references therein.

It has been argued recently that polarized current can affect the magnetic properties also of an homogeneous bulk ferromagnetic metal. [4, 5, 6, 7] Adding the spin torque linear in the spin current, to the Landau-Lifshitz equation of motion, one gets a modified spin-wave (SW) spectrum, which shows a current driven instability. In a half-metal, when the density of the minority carriers is zero, the spin current is equal to the electric current. In this case the uniform ferromagnetic state becomes unstable at a critical current given by the relation [4, 5]

$$
\mathbf{k} \cdot \mathbf{v}_{d}=\omega_{\mathbf{k}}
$$

where $\mathbf{k}$ is the spin-wave wave vector, $\omega_{\mathbf{k}}$ is the spin-wave dispersion, and $\mathbf{v}_{d}$ is the electron drift velocity, which is proportional to the electric current. This "Doppler-shift" critical current is of order of $10^{9} \mathrm{~A} / \mathrm{cm}^{2},[5]$ but in general, as has been shown by Tserkovnyak et al., 7] the critical current can be strongly enhanced.

High enough current densities can excite SW excitations in ferromagnetic layers even when the current is unpolarized, if the source and drain contacts are nonsymmetric. 8] SW excitation by an unpolarized current injected into a single ferromagnetic film from a point contact was observed by Ji et al. [9] and considered theoretically in Refs. 10 .

The original models by Slonczevski [2] and Berger [3] and all subsequent considerations of current induced SW excitation rely on the exchange model of interaction between the itinerant electrons and the localized spins. In this paper we concentrate on the electromagnetic (relativistic) interaction of the electron current with the field of the SW, which does not preserve the total spin. We show that in ferromagnetic conductors with large anomalous Hall effect this interaction can lead to current induced SW instability at critical unpolarized current of the same order or even smaller than that in the exchange interaction models. Unlike the exchange coupling of itinerant electrons with the SW, which is effective only in the vicinity of the interface between normal and ferromagnetic layers, [3] the above relativistic interaction acts also in the bulk of the ferromagnet. Therefore, the current induced SW instability cased by the Hall effect is not restricted to a layered structure of the ferromagnet.

The physical mechanism for SW generation via the relativistic interaction is as follows. Suppose for simplicity that an electric current $\mathbf{j}_{0}$ is driven parallel to the magnetization $\mathbf{M}_{0}$. Consider a spin wave propagating along $\mathbf{M}_{0}$. The oscillating magnetic moment and magnetic field of the spin wave lie in the plane perpendicular to $\mathbf{j}_{0}$ and $\mathbf{M}_{0}$. This gives rise to an alternating Hall current perpendicular to $\mathbf{M}_{0}$, which in his turn creates a magnetic field amplifying the field of the wave. If the electric field is strong enough, the amplification will exceed the damping due to eddy currents. If there are no other sources of damping, the spin-wave system becomes unstable at such field. We show in this paper that the instability is not restricted to the above simple geometry. It takes place at any mutual orientation of $\mathbf{j}_{0}$ and $\mathbf{M}_{0}$.

In ferromagnetic metals the main contribution to the Hall current comes from the anomalous Hall effect caused by the spin-orbit coupling in the metal. Since the anomalous Hall constant is by orders of magnitude larger than the normal one, the critical current for SW amplifying can be relatively small.

The full set of equations, which describes SW in a conducting media interacting with an electric current consists of the Landau-Lifsitz equation and of the Maxwell's 
equations. The Landau-Lifshitz equation for the precession of the magnetic moment is 11 ]

$$
\frac{\partial \mathbf{m}}{\partial t}=\gamma \mathbf{M}_{0} \times \mathbf{H}_{e f f}+\frac{1}{\tau_{2}} \mathbf{H}_{e f f}-\frac{1}{\tau_{1} M_{0}^{2}} \mathbf{M}_{0} \times\left(\mathbf{M}_{0} \times \mathbf{H}_{e f f}\right) .
$$

Here $\mathbf{m}$ is the (small) transverse alternative part of the magnetization, $m \ll M_{0}, \gamma$ is the gyromagnetic ratio, $\tau_{1}$ and $\tau_{2}$ are phenomenological SW relaxation times, and $\mathbf{H}_{\text {eff }}$ is the effective magnetic field given by

$$
\begin{array}{r}
\mathbf{H}_{e f f}=\mathbf{h}+\left[\frac{D}{\gamma M_{0}} \nabla^{2}-\frac{\mathbf{m}_{0} \cdot \mathbf{H}_{0}}{M_{0}}\right. \\
\left.-K\left(\mathbf{m}_{0} \cdot \mathbf{n}\right)^{2}\right] \mathbf{m}+K \mathbf{n}(\mathbf{n} \cdot \mathbf{m}),
\end{array}
$$

where $\mathbf{h}$ is the alternative part of the magnetic field, $D$ is the stiffness constant of the SW, $\mathbf{H}_{0}$ is the external magnetic field, which in restricted samples includes also the demagnetizing fields, $\mathbf{n}$ and $\mathbf{m}_{0}$ are unit vectors directed along the anisotropy axis and the magnetization respectively, and $K$ is the dimensionless anisotropy constant.

The Maxwell equations are:

$$
\begin{aligned}
\nabla \times \mathbf{h} & =\frac{4 \pi}{c} \mathbf{j} \\
\nabla \times \mathbf{e} & =-\frac{1}{c} \frac{\partial \mathbf{b}}{\partial t} \\
\nabla \cdot \mathbf{b} & =0
\end{aligned}
$$

Here the alternating magnetic induction is $\mathbf{b}=\mathbf{h}+4 \pi \mathbf{m}$, e is the alternating electric field, $c$ is the light velocity, and $\mathbf{j}$ is the alternating electric current given by [12]

$$
\mathbf{j}=\sigma \mathbf{e}+\sigma\left(R_{B} \mathbf{j}_{0} \times \mathbf{b}+R_{M} \mathbf{j}_{0} \times \mathbf{m}\right),
$$

where $R_{B}$ and $R_{M}$ are the ordinary and anomalous Hall coefficients respectively, $\sigma$ is the conductivity, and $\mathbf{j}_{0}$ is the dc part of the electric current density. We neglected in Eqs. (4) the displacement current, since for conductors considered here the inequality $\omega \ll \sigma$ always holds. We skipped in Eq. (5) a small term of order $\sigma\left(R_{B} B_{0}+R_{M} M_{0}\right) \ll 1$. The $\mathrm{SW}$ frequences considered in the paper are of order of $\left(10^{9}-10^{10}\right) s^{-1}$. i.e. much smaller than all typical electron relaxation frequencies in a ferromagnetic metal. Therefore, we used below the dc values of the transport coefficients.

The Maxwell equations, with the current from Eq. (5), relate the Fourier transforms of $\mathbf{h}$ and $\mathbf{m}$ as

$$
\begin{array}{r}
\mathbf{h}=-4 \pi\left[\omega_{0} k^{2}+i \frac{2}{\delta^{2}}\left(\mathbf{k} \cdot \mathbf{v}_{b}-\omega\right)\right]^{-1}\left[\omega_{0}(\mathbf{k} \cdot \mathbf{m}) \mathbf{k}\right. \\
\left.-i \frac{2}{\delta^{2}}(\mathbf{k} \cdot \mathbf{m}) \mathbf{v}_{m}-i \frac{2}{\delta^{2}}(\omega-\mathbf{k} \cdot \mathbf{v}) \mathbf{m}\right] .
\end{array}
$$

Here $\omega_{0}=4 \pi \gamma M_{0}$, the skin penetration depth, $\delta$, at frequency $\omega_{0}$ is given by $\delta=\left(c^{2} / 2 \pi \sigma \omega_{0}\right)^{1 / 2}$, and the effective velocities $\mathbf{v}_{b}, \mathbf{v}_{m}$ and $\mathbf{v}$ are related to the Hall coefficients by

$$
\mathbf{v}_{b}=R_{B} c \mathbf{j}_{0} \equiv \mathbf{v}_{d}, \quad \mathbf{v}_{m}=\frac{R_{M} c \mathbf{j}_{0}}{4 \pi}, \quad \mathbf{v}=\mathbf{v}_{b}+\mathbf{v}_{m}
$$

It is supposed that $\delta$ is much larger than the electron mean free path.

The Landau-Lifshitz equation yield another relation between $\mathbf{h}$ and $\mathbf{m}$. When $\mathbf{H}_{0}$ and $\mathbf{M}_{0}$ are parallel and directed along the anisotropy axis, this relation reads:

$$
\begin{aligned}
& \left(\Omega_{\mathbf{k}}-\omega-i \alpha \Omega_{\mathbf{k}}\right) m_{+}=\gamma M_{0} h_{+}(1-i \alpha) \\
& \left(\Omega_{\mathbf{k}}+\omega+i \alpha \Omega_{\mathbf{k}}\right) m_{-}=\gamma M_{0} h_{-}(1+i \alpha) .
\end{aligned}
$$

Here

$$
\alpha=\frac{1}{\gamma M_{0}}\left(\frac{1}{\tau_{1}}+\frac{1}{\tau_{2}}\right)
$$

$m_{ \pm}=m_{x} \pm i m_{y}, h_{ \pm}=h_{x} \pm i h_{y}$, while the axis $z$ is along the magnetization. The frequency $\Omega_{k}$ is given by

$$
\Omega_{k}=\gamma\left(H_{0}+H_{a}\right)+D k^{2},
$$

where $H_{a}$ is the anisotropy field: $H_{a}=K M_{0}$.

Eqs. (6) and (8) give the dispersion relation for SW in an external electric field. We consider in what follows wave-vectors $k$, which are larger than $\delta^{-1}: k \delta \gg 1$. One obtains then to leading order in the small parameter $1 / k^{2} \delta^{2}$ :

$$
\begin{array}{r}
\omega_{\mathbf{k}}^{2}\left(j_{0}\right)=\Omega_{\mathbf{k}} \Omega_{1 \mathbf{k}}+\frac{2 \omega_{\mathbf{k}}(0)}{\delta^{2} k^{2}}\left(\mathbf{k} \times \mathbf{v}_{m}\right) \cdot \mathbf{m}_{0} \\
-i\left[\frac{2}{\delta^{2} k^{2}}\left(\Omega_{1 \mathbf{k}}+\Omega_{\mathbf{k}} \cos ^{2} \theta\right)\left(\omega_{\mathbf{k}}(0)-\mathbf{k} \cdot \mathbf{v}\right)\right. \\
\left.+\alpha \omega_{\mathbf{k}}(0)\left(2 \Omega_{\mathbf{k}}+\omega_{0} \sin ^{2} \theta\right)\right] \\
-i \frac{2}{\delta^{2} k^{4}} \Omega_{\mathbf{k}} \mathbf{k} \cdot \mathbf{m}_{0}\left[\left(\mathbf{v}_{m} \cdot \mathbf{k}\right)\left(\mathbf{m}_{0} \cdot \mathbf{k}\right)-k^{2} \mathbf{v}_{m} \cdot \mathbf{m}_{0}\right]
\end{array}
$$

Here $\theta$ is the angle between the wave vector $\mathbf{k}$ and the magnetization, $\Omega_{1 \mathbf{k}}=\Omega_{\mathbf{k}}+\omega_{0} \sin ^{2} \theta$, and $\omega_{\mathbf{k}}(0) \equiv \omega_{\mathbf{k}}(\sigma=$ $0)=\sqrt{\Omega_{\mathbf{k}} \Omega_{1 \mathbf{k}}}$. In the absence of the current $j_{0}$, this equation gives the usual spectrum of SW decaying due to Landau-Lifshitz-Gilbert damping $\alpha$, and due to the eddy currents. The last decay is proportional to $1 / k^{2} \delta^{2}$.

In what follows we consider such wave vectors that the contribution to the SW damping from the last term in Eq. (11) is equal to zero. This happens specifically, if $\mathbf{k}$ is along or perpendicular to the magnetization, or at any $\theta$ provided $\mathbf{k}$ is along the current. Eq. (11) then yields:

$$
\begin{aligned}
& \operatorname{Re} \omega_{\mathbf{k}}\left(j_{0}\right)=\sqrt{\Omega_{\mathbf{k}} \Omega_{1 \mathbf{k}}}+\frac{\left(\mathbf{k} \times \mathbf{v}_{m}\right) \cdot \mathbf{m}_{0}}{\delta^{2} k^{2}} \\
& \operatorname{Im} \omega_{\mathbf{k}}\left(j_{0}\right)=-\left(\nu_{\mathbf{k}}+\alpha_{\mathbf{k}}\right)\left(\omega_{\mathbf{k}}(0)-\frac{\nu_{\mathbf{k}}}{\nu_{\mathbf{k}}+\alpha_{\mathbf{k}}} \mathbf{k} \cdot \mathbf{v}\right)
\end{aligned}
$$

Here

$$
\begin{aligned}
\nu_{\mathbf{k}} & =\frac{\Omega_{1 \mathbf{k}}+\Omega_{\mathbf{k}} \cos ^{2} \theta}{k^{2} \delta^{2} \sqrt{\Omega_{\mathbf{k}} \Omega_{1 \mathbf{k}}}} \\
\alpha_{\mathbf{k}} & =\frac{\alpha}{2 \sqrt{\Omega_{\mathbf{k}} \Omega_{1 \mathbf{k}}}}\left(2 \Omega_{\mathbf{k}}+\omega_{0} \sin ^{2} \theta\right) .
\end{aligned}
$$


Eq. (12) is our main result. It shows that $\operatorname{Im} \omega$ changes sign at a critical velocity, $\mathbf{v}_{c}$, given by the relation

$$
\mathbf{k} \cdot \mathbf{v}_{c}=\left(1+\frac{\alpha_{\mathbf{k}}}{\nu_{\mathbf{k}}}\right) \omega_{\mathbf{k}}(0) .
$$

At higher effective velocities, i.e. at higher currents the amplitude of spin waves, with $\mathbf{k}$ satisfying Eq (14), increases exponentially with time.

When $R_{M} / 4 \pi$ is smaller than $R_{B}$, i.e. $v=v_{b}=v_{d}$, the SW instability condition (14) resembles those, obtained in Refs. 4, 5, 6, 7 for SW instability in half-metals triggered by spin-transfer torques, see Eq. (11). However, $R_{M}$ in ferromagnetic conductors is usually by many orders of magnitude larger than $R_{B}$. We have, therefore, $v \approx v_{m} \gg v_{d}$, and the critical current for the instability considered here is much smaller than the critical current given by Eq (1).

Measurements [13] performed on $\mathrm{Fe}, \mathrm{Co}, \mathrm{Ni}$ and $\mathrm{Gd}$ films with the thickness of $1 \mu \mathrm{m}$, show that in pure metals, with the resistivity $\rho=\left(10^{-4}-10^{-6}\right) \Omega \mathrm{cm}$, the anomalous Hall conductivity $\sigma_{H}$ is of order $10^{3}(\Omega \mathrm{cm})^{-1}$. Thus, for metals with the resistivity $\rho=\left(10^{-4}-\right.$ $\left.10^{-5}\right) \Omega \mathrm{cm}$, and with $M_{0} \approx 10^{3} \mathrm{G}$, one gets $R_{M}=$ $\left(10^{-8}-10^{-10}\right) \Omega \mathrm{cm} / \mathrm{G}$. The values of $R_{M}$ for Ni films, which follow from the data obtained in Ref. 14, also fall in this region. It follows than from Eq (7) that the effective velocity $v_{m}$ is of order $v_{m}=\left(10^{-1}-10^{-3}\right) j_{0}$. Here $j_{0}$ is in $\mathrm{A} / \mathrm{cm}^{2}$, and $v_{m}$ in $\mathrm{cm} / \mathrm{sec}$. Note that the typical drift velocity is of order $10^{-4} j_{0} \mathrm{~cm} / \mathrm{sec}$. [5]

The real part of the SW frequency, Eq. (12), also acquires a term linear in the current, which is solely caused by the anomalous Hall effect. The frequency of spin waves, with $\mathbf{k}$ non-parallel to the magnetization and to the current, is modified by the current. The current increases or decreases the frequency, depending on the direction of $\mathbf{k}$.

When the wave-vector $\mathbf{k}$ and the current are along the magnetization, the critical velocity is given by

$$
v_{c}(k)=\left[D k+\frac{\gamma\left(H_{0}+H_{a}\right)}{k}\right]\left(1+\frac{\alpha}{2} \delta^{2} k^{2}\right) .
$$

$v_{c}(k)$ is minimal at $k=k_{0}$ given by

$$
k_{0}^{2}=\frac{1}{6 \alpha \delta^{2}}\left[-(2+\alpha \beta)+\sqrt{4+28 \alpha \beta+(\alpha \beta)^{2}}\right],
$$

where $\beta=\gamma\left(H_{0}+H_{a}\right) \delta^{2} / D$.

When the damping $\alpha$ is small, $\alpha \ll 1 / \beta$, the critical velocity coincides with the phase velocity of the $\mathrm{SW}, 12$ and $k_{0}$ is given by $k_{0}=\left[\gamma\left(H_{0}+H_{a}\right) / D\right]^{1 / 2}, v_{c}\left(k_{0}\right)$ being equal to

$$
v_{c}\left(k_{0}\right)=2 \sqrt{D \gamma\left(H_{0}+H_{a}\right)} .
$$

With typical values $D=(0.1-0.05) \mathrm{cm}^{2} / \mathrm{sec}$ and $\gamma\left(H_{0}+H_{a}\right)=2 \cdot 10^{9} \mathrm{sec}^{-1}$, one gets $v_{c}\left(k_{0}\right) \approx 3 \cdot 10^{4}$

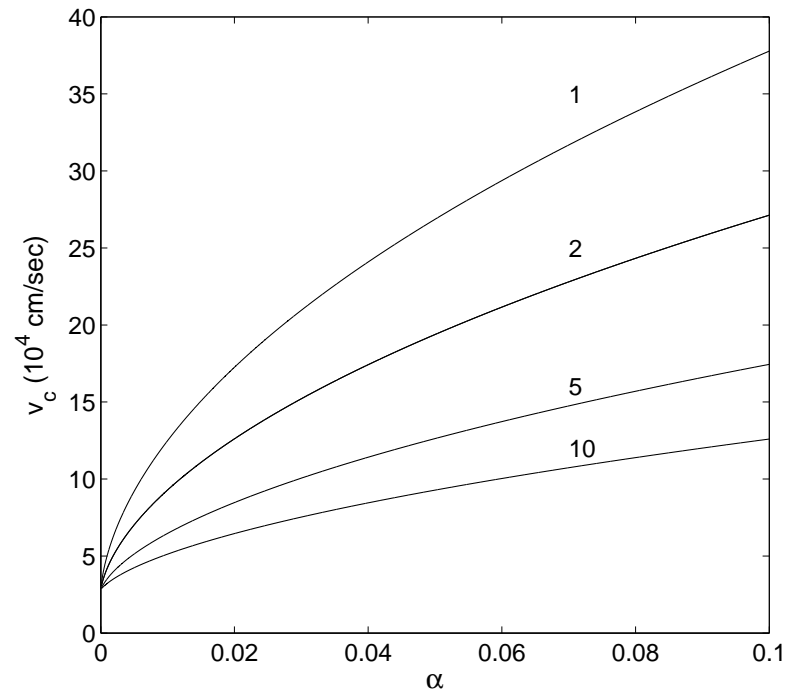

FIG. 1: Dependence of the minimal critical velocity on $\alpha$ at different conductivities $\sigma=(1,2,5,10) \times 10^{4}(\Omega \mathrm{cm})^{-1}$, with parameters $D=0.1 \mathrm{~cm}^{2} / \mathrm{sec}, H_{0}+H_{a}=100$ Oe. Both $\mathbf{k}$ and $\mathbf{j}_{0}$ are along the magnetization.

$\mathrm{cm} / \mathrm{sec}$. Thus, the minimal critical current density is $j_{c}=4 \pi v_{c}\left(k_{0}\right) / R_{M} c \approx\left(3 \cdot 10^{5}-3 \cdot 10^{7}\right) \mathrm{A} / \mathrm{cm}^{2}$. This value of $j_{c}$ is by several orders of magnitude smaller than that obtained in Refs $\underline{5}$ and $\underline{6}$.

The wave vector $k_{0}$ decreases with increase of $\alpha$. If $\alpha$ is large, $\alpha \gg 1 / \beta$, it follows from Eq. (16), that $k_{0}$ does not depend on $D$ and $\gamma H_{0}$, and is equal to: $k_{0}=\left(2 / \alpha \delta^{2}\right)^{1 / 2}$. The critical velocity in this case is

$$
v_{c}\left(k_{0}\right)=\delta \gamma\left(H_{0}+H_{a}\right) \sqrt{2 \alpha},
$$

increasing linearly with the external magnetic field.

With $\sigma=10^{5} \mathrm{Om}^{-1} \cdot \mathrm{cm}^{-1}$, and $\omega_{0}=10^{11} \mathrm{sec}^{-1}$, one gets $\delta=10^{-4} \mathrm{~cm}$. Then, with the above values of $D$ and $\gamma\left(H_{0}+H_{a}\right)$ one finds that the inequality $\alpha \gg 1 / \beta$ is fulfilled, if $\alpha$ is larger than $10^{-2}$. The dependence of the minimal critical velocity on $\alpha$ at different values of the conductivity is shown in Fig. 1.

If $\mathbf{k}$ is parallel to the current and perpendicular to the magnetization, Eqs. (14) and (13) yield

$$
\left.v_{c}(k)=\frac{1}{k} \sqrt{\Omega_{k}\left(\Omega_{k}+\omega_{0}\right.}\right)\left(1+\frac{\alpha \delta^{2} k^{2}}{2} \frac{2 \Omega_{k}+\omega_{0}}{\Omega_{k}+\omega_{0}}\right) .
$$

Usually the inequality $\gamma H_{0} \ll \omega_{0}$ holds. Then, at small $\alpha, \alpha \ll 1 / \beta$, the minimal critical velocity is

$$
v_{c}\left(k_{0}\right)=\sqrt{D \omega_{0}} \approx 10^{5} \mathrm{~cm} / \mathrm{sec},
$$

while the minimal critical current is of order $\left(10^{6}-10^{8}\right)$ $\mathrm{A} / \mathrm{cm}^{2}$. As before, the critical velocity increases with increase of $\alpha$. When $\alpha$ is large, and satisfies the inequality $\alpha \gg 1 / \beta$, the minimal critical velocity is

$$
v_{c}\left(k_{0}\right)=2 \delta \sqrt{\gamma\left(H_{0}+H_{a}\right) \omega_{0} \alpha},
$$


The critical velocity and the critical current can be considerably smaller than the above values, if the ferromagnet is in a state close to an orientational phase transition caused by an external magnetic field. We consider now the instability condition in several different arrangements of this type. In all cases we suppose that $\mathbf{k}$ is along the dc current, since we are interested in the minimal critical current.

First, let in a uniaxial ferromagnet the external magnetic field is aligned perpendicular to the easy axis, and $H_{0}$ is larger but close to $H_{a}$. Then, the magnetization points along $\mathbf{H}_{0}$. Repeating the previous calculation for this orientation of the field, one gets for $\mathbf{k}$ and $\mathbf{j}_{0}$ along the magnetization:

$$
v_{c}(k)=\frac{\omega_{k}}{k}\left(1+\frac{\alpha \delta^{2} k^{2}}{2}\right),
$$

where [1]

$$
\omega_{\mathbf{k}}=\sqrt{\left[D k^{2}+\gamma\left(H_{0}-H_{a}\right)\right]\left(D k^{2}+\gamma H_{0}\right)} .
$$

At small $\alpha$

$$
\alpha \ll \frac{D}{\gamma\left|H_{0}-H_{a}\right| \delta^{2}},
$$

and at $H_{0}-H_{a} \ll H_{a}$, the critical velocity is minimum at a wave vector, given by

$$
k_{0}=\left[\frac{\gamma^{2} H_{a}\left(H_{0}-H_{a}\right)}{D\left(D+\alpha \delta^{2} \gamma H_{a}\right)}\right]^{1 / 4},
$$

and $v_{c}\left(k_{0}\right)$ is

$$
v_{c}\left(k_{0}\right)=\sqrt{D \gamma H_{a}}
$$

which is considerably smaller than the critical velocity (17). At large $\alpha$ : $\alpha \gg D / \gamma\left(H_{0}-H_{a}\right) \delta^{2}$, the critical velocity equals to

$$
v_{c}=\gamma \delta \sqrt{2 \alpha H_{a}\left(H_{0}-H_{a}\right)} .
$$

Note that if $\left(H_{0}-H_{a}\right) / H_{a}$ is small, the inequalities Eq. (24) and $k_{0}^{2} \delta^{2} \ll 1$ are fulfilled whenever $\alpha$ is smaller than 1, i.e. the SW damping in this case almost does not affect the critical current.

Suppose now that the ferromagnet is in a metastable state, with the field $\mathbf{H}_{0}$ smaller than $H_{a}$ and opposite in direction to the magnetization. It follows than from Eq. (3) that in Eq. (15) and in all subsequent equations for $v_{c}$ the field $H_{0}$ should be replaced by $-H_{0}$. Hence, when $H_{0}$ approaches $H_{a}$ the critical wave-vector and $v_{c}$ tend to zero. $k_{0}$ is restricted from below by the inequality $k_{0}^{2} \delta^{2} \gg 1$. This gives for small damping $\alpha$, which satisfies the inequality (24): $\gamma\left(H_{a}-H_{0}\right) \gg D \delta^{-2}, v_{c} \gg 2 D / \delta \approx$ $10^{3} \mathrm{~cm} / \mathrm{sec}$, and $j_{c} \gg 10^{4} \mathrm{~A} / \mathrm{cm}^{2}$. This implies that a relatively small current of order or larger than $(3-$ 5) $\times 10^{4} \mathrm{~A} / \mathrm{cm}^{2}$ can drive the magnetization switching at magnetic fields smaller than $H_{a}$, if the current flows along the magnetization. Note that, as in the previous case, the inequality Eq. (24) is equivalent to the condition $\alpha \ll 1$.

Finally, consider thin films, with $k d \ll 1, d$ is the film width. The SW spectrum in this case at different directions of the magnetization and external magnetic field was derived in many papers, see e.g. Refs. 15. We consider the case, when the external magnetic field is perpendicular to the film plane and larger than $4 \pi M_{0}+H_{s}-H_{a}$, where $H_{a}$ is the volume anisotropy field, the easy axis being in a symmetry direction of the film, and $H_{s}$ is the surface anisotropy field. Then, the magnetization is also perpendicular to the film, and the spectrum of SW with $\mathbf{k}$ in the plane is:

$$
\omega_{k}=\sqrt{\left[\gamma\left(\tilde{H}+2 \pi M_{0} k d\right)+D k^{2}\right]\left[\gamma \tilde{H}+D k^{2}\right]}
$$

where $\tilde{H}=H_{0}+H_{a}-H_{s}-4 \pi M_{0}$.

As argued above, the damping can be neglected if the ferromagnet is in the vicinity of the phase transition. Then the critical velocity is equal to the SW phase velocity, and is minimal at $k_{0}=\sqrt{\gamma \tilde{H} / D}$, while $\tilde{H}$ should be larger than $D / \gamma \delta^{2}$. The minimal critical velocity is given by:

$$
v_{c}\left(k_{0}\right)=\left(4 \gamma \tilde{H} D+\omega_{0} d \sqrt{\gamma \tilde{H} D}\right)^{1 / 2} .
$$

The above inequalities yield that $v_{c}\left(k_{0}\right)$ is restricted from below as: $v_{c}>\sqrt{\omega_{0} D d / \delta} \approx 10^{4} \mathrm{~cm} / \mathrm{sec}$. Hence, the critical current is larger than $\left(10^{5}-10^{7}\right) \mathrm{A} / \mathrm{cm}^{2}$.

In conclusion, we have calculated the effect of an electric current on the SW spectrum in a ferromagnetic metal. We have shown that the ordinary and anomalous Hall currents lead to the reduction of the SW damping, caused by the eddy currents. At sufficiently strong currents the damping changes sign, and a SW instability develops. The critical current of the instability is determined mainly by the anomalous Hall effect, and may be much smaller than the critical current for SW excitation with spin-transfer torques.

The author thanks A. Gerber for helpful discussions. This research was supported by the Israel Science Foundation grant No. 633/06.

[1] Y. Tserkovnyak, A. Brataas, G. E. W. Bauer, and B. I. Halperin, Rev. Mod. Phys. 77, 1375 (2005).

[2] J. C. Slonczewski, J. Magn. Magn. Mater 159, L1 (1996); J. C. Slonczewski, J. Magn. Magn. Mater 195, L261 (1999).

[3] L. Berger, Phys. Rev. B 54, 9353 (1996)

[4] Ya. B. Bazaliy, B. A. Jones, and S.-C. Zhang, Phys. Rev. B 57, R3213 (1998).

[5] J. Fernández-Rossier, M. Braun, A. S. Núñez, and A. H. MacDonald, Phys. Rev. B 69, 174412 (2004). 
[6] Z. Li and S.Zhang, Phys. Rev. Lett. 92, 207203 (2004).

[7] Y. Tserkovnyak, H. J. Skadsem, A. Brataas, and G. E. W. Bauer, Phys. Rev. B 74, 144405 (2006).

[8] M. L. Polianski and P. W. Brouwer, Phys. Rev. Lett. 92, 026602 (2004).

[9] Y. Ji, C. L. Chien, and M. D. Stiles, Phys. Rev. Lett. 90, 106601 (2003). M. D. Stiles, J. Xiao and A. Zangwill, Phys. Rev. B 69, 054408 (2004).

[10] H. Xi, Y. Yang, J. Ouyang, Y. Shi, and K.-Z. Gao, Phys. Rev. B 75, 174411 (2007).

[11] A. I. Akhiezer, V. G. Bar'yakhtar, and S. V. Peletminskii, Spin Waves (North-Holland, Amsterdam, 1969).
[12] L. É Gurevich and I. Ya. Korenblit, Zh. Eksp. Teor. Fiz. 48, 652 (1965) [Sov. Phys. JETP 21, 431 (1965)].

[13] T. Miyasato, N. Abe, T. Fujii, A. Asamitsu, S. Onoda, Y. Onose, N. Nagaosa, and Y. Tokura, Phys. Rev. Lett. 99, 086602 (2007).

[14] A. Gerber, A. Milner, A. Finkler, M. Karpovski, L. Goldsmith, J. Tuaillon-Combes, O. Boisron, P. Mélinon, and A. Perez, Phys. Rev. B 69, 224403 (2004).

[15] R. Arias and D. L. Mills, Phys. Rev. B 60, 7395 (1999); S. M. Rezende, F. M. de Aguiar, and A. Azevedo, Phys. Rev. B 73, 094402 (2006). 